\title{
Commentaire
}

\section{Les consultations en personne pour les victimes de violence conjugale : un service que les spécialistes en soins chroniques doivent considérer comme essentiel}

\author{
Marzieh Eghtesadi, M.D.
}

Publié en ligne le 10 mars 2021

Diffuser cet article sur Twitter

Les activités cliniques entourant les maladies qui n'engagent pas le pronostic vital de la personne ont été jugées non essentielles, afin d'endiguer l'épidémie de la COVID-19. Le personnel infirmier et paramédical a été transféré vers des établissements de soins actifs et les consultations ont été suspendues dans la plupart des secteurs qui traitent les maladies chroniques. En dépit des difficultés initiales liées à la mise en place des télécommunications me permettant, en tant que médecin spécialisée dans les céphalées, d’offrir des soins de santé à distance, la plupart de mes patients ont été reconnaissants de cet accès à la télésanté. Celle-ci leur a permis de maintenir un contact médical avec moi en toute sécurité.

Cependant, j'ai observé que ce nouveau modèle de soins ne servait pas au mieux les intérêts de tous mes patients.

Les effets néfastes du confinement sur les populations de patients vulnérables n'ayant pas accès au soutien collectif des réseaux sociaux suscitent une inquiétude généralisée ${ }^{1}$. Plus particulièrement, les victimes de violence conjugale ont fait état d'un risque accru de violence ${ }^{2}$. Étant donné que je m'occupe de patients qui non seulement sont majoritairement des femmes, mais ont également une hypersensibilité cérébrale innée aux stimuli aversifs, on me confie souvent des informations sensibles sur les déclencheurs de céphalées, dont la détresse émotionnelle causée par la violence conjugale ${ }^{3}$.

Les directives sur les mesures d'urgence associées à la pandémie de COVID-19 n'ont pas autorisé les consultations médicales en personne pour les victimes de violence conjugale. Les consultations en personne ont été limitées aux examens physiques dans le cas d'une affection aiguë potentiellement fatale, comme une céphalée en coup de tonnerre évoquant un saignement intracrânien, ou les dysfonctionnements de dispositifs thérapeutiques perfectionnés, comme les systèmes d'administration de médicaments parentéraux et les modulateurs implantables de signalisation nerveuse $^{4,5}$. Heureusement pour mes patients subissant de la violence conjugale, la direction de mon hôpital a accédé à ma demande de maintenir les consultations médicales en personne, quel que soit l'état de la céphalée. Bien entendu, un dépistage des symptômes de COVID-19 est effectué 24 heures ou moins avant le rendez-vous et les mesures de sécurité (en particulier hygiène des mains, port du masque et distanciation physique) sont appliquées.

Ce n'est pas parce qu'un service est offert pour un motif non urgent ou qui n'engage pas le pronostic vital que ce service n'est pas essentiel. Plusieurs facteurs sont susceptibles de restreindre l'accès aux soins de santé des victimes de violence conjugale : les coûts, la crainte des préjugés et de la discrimination, les tactiques de contrôle des partenaires violents et un faible sentiment d'efficacité personnelle $e^{6-8}$. Les mesures d'urgence qui ont dû être prises pour freiner la pandémie ont accentué la vulnérabilité à la violence conjugale, en raison des crises créées par les pertes et suppressions d'emplois et en raison également de l'isolement social dû la distanciation physique obligatoire.
Malgré l'augmentation anticipée du nombre d'incidents en lien avec la violence conjugale, les services d'intervention d'urgence ont constaté une diminution du nombre de victimes qui demandent de l'aide, probablement en raison de leur crainte de s'exposer et d'exposer leurs proches au virus et également car elles se retrouvent coincées avec leur agresseur?

La pandémie a également réduit l'accès à des lieux sûrs. La plupart des refuges fonctionnent à moindre capacité, les déplacements sont restreints, le transport en commun a été réduit et des couvre-feux ont été imposés ${ }^{10}$. De plus, comme la majorité des centres de soins n'autorisent plus les consultations sans rendez-vous, les victimes sont susceptibles d'être menacées lorsqu'elles prennent rendez-vous en ligne ou par téléphone en présence de leur agresseur $^{11}$. En outre, les victimes de violence conjugale qui appartiennent à des communautés ethniques marginalisées sont susceptibles d'être davantage méfiantes vis-à-vis des autorités policières, du fait que les policiers infligent des sanctions financières en cas de non-respect du confinement, et ces perceptions négatives du système judiciaire se traduisent souvent par une méfiance à l'égard des institutions médicales ${ }^{12,13}$.

Les activités cliniques en santé sexuelle et reproductive ont été maintenues pour les victimes de violence familiale, afin de repérer les dommages collatéraux de l'isolement social attribuable à la pandémie ${ }^{14}$. Toutefois, si l'accès à son fournisseur de soins chroniques est limité, une patiente victime de violence conjugale risque de ne 
pas vouloir se confier pour obtenir de l'aide à un professionnel qu'elle ne connaît pas. Elle peut craindre par exemple un rendez-vous précipité et un manque de compréhension des professionnels dans les établissements de soins aigus. De plus, elle peut se sentir coupable d'accaparer le personnel des services d'urgence, déjà au maximum de leur capacité. Elle peut se sentir mal à l'aise à l'idée d'aborder des sujets sensibles dans un cadre inconnu, surtout sur un brancard dans le couloir ou au sein d'un service d'urgence bruyant où la confidentialité est limitée.

Les médecins de tous les secteurs offrant des soins aux malades chroniques devraient être encouragés à considérer leurs services comme essentiels si leurs patients risquent d'être victimes de violence en raison des mesures de confinement en place. La gestion des problèmes de santé chroniques implique un suivi à long terme, souvent sur plusieurs années, ce qui crée une alliance thérapeutique entre le médecin et son patient et peut inciter ce dernier à demander de l'aide en temps de crise. Cette alliance est généralement le reflet de la qualité de la communication entre le patient et le fournisseur de soins de santé, il suppose une relation personnelle et un travail de collaboration ${ }^{15}$.

La relation entre patient et médecin étant au cœur de leur rôle, les médecins de famille établissent souvent des alliances de ce type avec leurs patients, grâce à une coordination globale des soins qui ne se limite pas à la maladie ${ }^{16,17}$. Les alliances entre patient et professionnel les plus fréquentes, hors contexte de la santé sexuelle ou reproductive, relèvent par exemple du traitement à long terme des endocrinopathies, des maladies auto-immunes ou inflammatoires, des maladies rénales chroniques et des affections respiratoires ou cardiaques ${ }^{15}$. L'approche que nous proposons ici est susceptible de se révéler utile dans le contexte d'une relation déjà en place avec une patiente ayant confié des antécédents de violence et d'abus.

Dans le cadre de la restructuration des soins de santé pendant la pandémie, les médecins qui connaissent des patients ayant été victimes de violence familiale devraient, dans la mesure où la logistique le permet, prévoir au moins une consultation en personne avec chacun d'eux. La plupart des médecins ont sans doute déjà abordé la possibilité pour leurs patients d'obtenir de l'aide mais, souvent, ces discussions demeurent superficielles et se limitent à encourager la personne à recourir aux ressources communautaires, sans compter que de nombreux médecins doivent composer avec une lourde charge de travail et ne ressentent en eux pas la confiance nécessaire pour aider ces patients ${ }^{6}$. Pourtant, les interventions répétées d'un médecin en qui le patient a confiance, dans un environnement sûr, sont opportunes et perçues favorablement par le patient, à la différence des appels téléphoniques de suivi ${ }^{18}$.

Dans ce contexte, la consultation en personne ne vise pas à déceler la violence conjugale, car la patiente a déjà divulgué cette information, mais plutôt à évaluer sa maladie chronique et à lui offrir d'être mis en relation avec des ressources spécialisées en violence conjugale durant l'épidémie et la crise sanitaire psychosociale. Si la personne a besoin d'un plan de protection, le médecin doit demeurer conscient des limites de son expertise en matière de violence conjugale et juger de la pertinence de communiquer avec d'autres professionnels.

Depuis le début de la pandémie de COVID19, de nombreux établissements ont mis en place une équipe de soutien social en cas de crise, que les travailleurs de la santé qui ont besoin de conseils et d'expertise peuvent consulter pour aider leurs patients. Voici quelques conseils pratiques et des exemples de points à aborder pendant l'évaluation en personne avec une victime de violence conjugale :

- Pour vos patients qui maîtrisent mal la technologie ou qui ont un accès limité à un ordinateur, prévoyez du temps pendant la rencontre pour leur fournir l'adresse de l'hôpital et des refuges les plus proches de leur domicile ainsi que d'autres ressources telles que les centres culturels communautaires. Imprimez leur au besoin des cartes de ces emplacements. Veillez à leur fournir des données à jour sur ces ressources et à leur indiquer tout changement opérationnel/organisationnel en lien avec la pandémie.

- Lors de la planification du prochain rendez-vous de suivi, demandez à votre patiente quel est le meilleur moment pour l'appeler afin qu'elle soit seule pour discuter de ses problèmes de santé.
- Dans les établissements où les notes médicales sont encore manuscrites, assurez-vous que le scénario de protection établi avec votre patiente soit retranscrit en format électronique et compréhensible pour tout professionnel de la santé au cas où elle doive se présenter aux urgences. Cela permet aussi de garantir à la patiente qu'elle n'aura pas à fournir de longues explications lors du triage, surtout si son agresseur l'accompagne.

- Précisez à votre patiente la date et l'endroit exact dans les dossiers où le scénario de protection est classé. Elle pourra ainsi le signaler discrètement et rapidement au personnel de triage.

- Vérifiez la liste des rendez-vous annulés et celle des patients qui ne se sont pas présentés à un rendez-vous, afin de vous assurer que ces derniers n'ont pas été retirés de la liste d'attente à votre insu. En effet, certains établissements autorisent un nombre limité d'annulations, de rendez-vous manqués ou de tentatives de report, à la suite de quoi ils suppriment automatiquement la personne des listes.

- Évitez de décrire ces patientes comme victimes de violence conjugale à votre personnel administratif. De nombreuses personnes concernées par cette situation ne désirent pas ce type d'étiquette, et de multiples raisons peuvent justifier par ailleurs une consultation en personne, comme la nécessité d'un examen physique si on décèle un signal d'alarme lors d'un entretien en télésanté (comme, en neurologie, une perte de vision transitoire accompagnée d'un mal de tête). Il n'est donc pas nécessaire de préciser au personnel non médical et au personnel non impliqué dans le plan de soins d'un patient la raison d'une visite confidentielle.

Après cette consultation en personne, le médecin va pouvoir décider de la fréquence du suivi médical et de ses modalités. Toutefois, si le maintien du contact avec une patiente relève du contexte de violence familiale, il serait pertinent d'orienter celle-ci vers des experts en violence conjugale, plus à même de faire valoir la nécessité de soins. Si la patiente et le médecin préfèrent poursuivre les soins en télésanté, le médecin peut envoyer une lettre à la patiente avec une date provisoire de rendez-vous en personne après la pandémie. La patiente peut placer cette lettre bien en vue, pour rappeler à tous les 
membres du foyer qu'elle est suivie par un professionnel de la santé qui compte évaluer son bien-être et procéder à un examen après la pandémie.

Selon Mechanic et Schlesinger ${ }^{19}$, « le succès des soins médicaux dépend avant tout de la confiance des patients envers le fait que leurs médecins [...] accordent la plus haute priorité au bien-être de leurs patients ». Comme il faudra probablement du temps aux autorités de santé publique pour mettre en place des initiatives visant à réduire les répercussions sexospécifiques de la pandémie de COVID-19, nous devons veiller à ce que nos populations de patients vulnérables ne soient pas négligées ${ }^{20}$. Malgré les défis éthiques posés par les mesures d'urgence liées à la COVID-19 et la recherche d'un équilibre entre sécurité publique et soins axés sur le patient, nous devons reconnaître les situations où nous sommes appelés à prendre la défense de nos patients, en particulier de ceux qui ne le font pas ou ne sont pas en état de le faire. En tant que fournisseurs de soins de première ligne, nous devons exposer notre point de vue aux autorités de santé publique, notamment en ce qui concerne les facteurs principaux susceptibles d'influencer la décision d'offrir des consultations en personne pendant la pandémie. Ces facteurs vont souvent au-delà des questions pratiques habituelles sur la nécessité d'un examen physique ou la facilité d'accès d'un patient à la technologie requise pour les soins virtuels.

Dans cet article, je tiens à souligner l'importance de maintenir un contact médical, en particulier une consultation en personne dans la mesure du possible, avec les victimes de violence conjugale ayant déjà révélé subir des actes violents. Ce contact est particulièrement précieux lorsqu'une alliance thérapeutique a été établie avec une patiente, par exemple au fil de la prise en charge d'une maladie chronique. Bien que cet article soit rédigé du point de vue d'une pratique en médecine des céphalées, les questions qu'il soulève sont applicables plus largement, au-delà de la spécialité de la clinique, de la répartition par sexe des patients ou du manque de personnel dû au redéploiement occasionné par la COVID-19. Les médecins qui travaillent dans les secteurs non essentiels à l'intervention directe contre la COVID-19 et dont les activités cliniques ont été modifiées devraient envisager de maintenir leurs services en tant que soins essentiels pour ce groupe de patients.

Comme de nombreux services sociaux communautaires enregistrent une augmentation importante des délais d'attente, il est important de rester flexible envers nos patients. En outre, le médecin traitant du patient doit participer, si le patient y consent, au plan de soins. Il n'est cependant pas rare que le clinicien responsable du traitement d'une maladie chronique, en particulier en cas de maladies complexes de niveau tertiaire, ait une alliance thérapeutique plus forte avec le patient en raison des contacts médicaux fréquents que cela implique.

Enfin, dans le cadre de l'amélioration souhaitée par les autorités de santé publique en matière de restructuration des soins de santé durant la pandémie, les médecins sont invités à collaborer avec d'autres équipes institutionnelles spécialisées lorsque leur expertise s'avère limitée, en particulier avec le personnel psychosocial, dans le but d'aider les populations marginalisées et vulnérables. Cette collaboration peut s'avérer particulièrement profitable aux victimes de violence conjugale, dans la mesure où un scénario de protection bien conçu tient compte de la dynamique complexe des situations d'abus, au-delà de l'expérience de la violence.

\section{Remerciements}

La $D^{\text {re }}$ Eghtesadi est lauréate du Prix de l'innovation 2020 de l'Université de Montréal pour ses travaux sur la santé des femmes et l'accès aux soins des céphalées.

\section{Financement}

Aucun

\section{Conflits d'intérêts}

Aucun

\section{Contribution et avis}

La $\mathrm{D}^{\text {re }}$ Eghtesadi a rédigé le manuscrit et approuvé la version finale de ce dernier et elle accepte la responsabilité de son exactitude et de son intégrité.

Le contenu de l'article et les points de vue qui y sont exprimés n'engagent que l'auteure; ils ne correspondent pas nécessairement à ceux du gouvernement du Canada.

\section{Références}

1. Brooks S.K., R.K. Webster, L.E. Smith et collab. The psychological impact of quarantine and how to reduce it: rapid review of the evidence. Lancet. 2020; 395(10227):912-920. https://doi.org /10.1016/S0140-6736(20)30460-8
2. Anurudran A., L. Yared, C. Comrie, K. Harrison, T. Burke. Domestic violence amid COVID-19. Int J Gynaecol Obstet. 2020;150(2):255-256. https://doi.org /10.1002/ijgo.13247

3. Wilcox S.L., R. Veggeberg, J. Lemme et collab. Increased functional activation of limbic brain regions during negative emotional processing in migraine. Front Hum Neurosci. 2016;10:366. https://doi.org/10.3389/fnhum.2016 .00366

4. Dodick D.W. Clinical clues and clinical rules: primary vs secondary headache. Adv Stud Med. 2003;3(6C): S550-555.

5. Eghtesadi M., E. Leroux, M.-P. FournierGosselin et collab. Neurostimulation for refractory cervicogenic headache: a three-year retrospective study. Neuromodulation. 2018;21(3):302-309. https://doi.org/10.1111/ner.12730

6. Rodríguez M., J.M. Valentine, J.B. Son, M. Muhammad. Intimate partner violence and barriers to mental health care for ethnically diverse populations of women. Trauma Violence Abuse. 2009;10(4):358-374. https://doi.org/10 $.1177 / 1524838009339756$

7. Moreira D.N., M. Pinto da Costa. The impact of the Covid-19 pandemic in the precipitation of intimate partner violence. Int J Law Psychiatry. 2020; 71:101606. https://doi.org/10.1016/j .ijlp.2020.101606

8. Wilson K.S., M.R. Silberberg, A.J. Brown, S.D. Yaggy. Health needs and barriers to healthcare of women who have experienced intimate partner violence. J Women Health. 2007;16(10): 1485-1498. https://doi.org/10.1089/jwh .2007 .0385

9. Smith C. Decline in domestic violence calls during COVID-19 a sad situation, Moncton agency warns. Global News. 5 mai 2020 (consultation le 1er janvier 2021). En ligne à : https://globalnews .ca/news/6908469/domestic-violence -concerns-covid-19/

10. Evans ML., M. Lindauer, ME Farrell. A pandemic within a pandemic-intimate partner violence during Covid-19. N Engl J Med. 2020;383(24):2302-2304. https://doi.org/10.1056/NEJMp2024046 
11. Women's Aid. The impact of COVID-19 on women and children experiencing domestic abuse, and the life-saving services that support them. Bristol (UK) : Women's Aid; 2020 (consultation le 1er janvier 2021). En ligne à : https://www.womensaid.org.uk /the-impact-of-covid-19-on-women -and-children-experiencing-domestic -abuse-and-the-life-saving-services -that-support-them/

12. Alang S., DD. McAlpine, R. Hardeman. Police brutality and mistrust in medical institutions. J Racial Ethn Health Disparities. 2020;7(4):760-768. https:// doi.org/10.1007/s40615-020-00706-w

13. Laurencin CT., JM. Walker. A pandemic on a pandemic: racism and COVID19 in Blacks. Cell Syst. 2020;11(1):9-10. https://doi.org/10.1016/j.cels.2020.07 .002

14. Hall K.S., G. Samari, S. Garbers et collab. Centring sexual and reproductive health and justice in the global COVID-19 response. Lancet. 2020; 395(10231):1175-1177. https://doi.org /10.1016/S0140-6736(20)30801-1

15. Allen M.L., B.L. Cook, N. Carson, A. Interian, M. La Roche, M. Alegría. Patient-provider therapeutic alliance contributes to patient activation in community mental health clinics. Adm Policy Ment Health. 2017;44(4):431440. https://doi.org/10.1007/s10488 $-015-0655-8$

16. Novack D.H. Novack DH. Therapeutic aspects of the clinical encounter. Dans : Lipkin MJ, Putnam SM, Lazare A, et al. (dir.). The medical interview. New York: Springer-Verlag; 1995. p. 32-49.

17. Shaw E, Oandasan I, Fowler N (dir.). CanMEDS-MF 2017 : Un référentiel de compétences pour les médecins de famille dans tout le continuum de formation. Mississauga (ON) : Collège des médecins de famille du Canada; 2017.

18. Feder G.S., M. Hutson, J. Ramsay, A.R. Taket. Women exposed to intimate partner violence: expectations and experiences when they encounter health care professionals: a meta-analysis of qualitative studies. Arch Intern Med. 2006;166(1):22-37. https://doi.org/10 $.1001 /$ archinte.166.1.22
19. Mechanic D., M. Schlesinger. The impact of managed care on patients' trust in medical care and their physicians. JAMA. 1996;275(21):1693-1697. https://doi.org/10.1001/jama.1996.0353 0450083048

20. Wenham C., J. Smith, R. Morgan; Gender and COVID-19 Working Group. COVID-19: the gendered impacts of the outbreak. Lancet. 2020;395(10227): 846-848. https://doi.org/10.1016/S0140 $-6736(20) 30526-2$ 